\title{
Validation of PeneloPET Against Two Small Animal PET Scanners
}

\author{
S. España, J. L. Herraiz, E. Vicente, E. Herranz, J. J. Vaquero, M. Desco, and J. M. Udias
}

\begin{abstract}
PeneloPET is a Monte Carlo application based on PENELOPE. We present here the new features and results of validation tests for the new version of PeneloPET that has been compared against data from real scanners. PeneloPET was built as a powerful tool for PET simulation, it is easy to use, fast and very accurate. Recently, many improvements have been made in the code with the incorporation of a very realistic signal processing chain and by adding the possibility of running simulations in parallel mode on cluster computers. A comparison between data obtained with two small-animal scanners and the results of PeneloPET simulations has been performed. The smallanimal PET scanners were an eXplore Vista DR (GEHC) and a partial-ring, rotating rPET (SUINSA Medical Systems). Intrinsic resolution, scatter fractions, noise equivalent count rates and sensitivity measurements for the real acquisitions and simulations were compared. NEMA protocol was applied using mouse-size and rat-size cylinders, spheres and line sources as phantoms. Results show small differences (less than 10\%) between real acquisitions and simulated data, proving that PeneloPET is an accurate tool for PET simulations.
\end{abstract}

\section{INTRODUCTION}

$\mathrm{N}_{\mathrm{r} e \mathrm{sen}}^{\mathrm{o}}$ OWADAYS, Monte Carlo simulations are a basic tool for research and development in PET. PET dedicated simulators such as SimSET [1] and GATE [2] are extensively used these days.

PeneloPET [3] is a Monte Carlo application based on PENELOPE [4] to perform general simulations in PET. It was built as a powerful tool for PET simulations, easy to use, fast and with very accurate results. An extensive validation of PeneloPET by comparison to real data from two small animal scanners is presented here. The small animal PET scanners employed were the eXplore Vista DR (GEHC) [5] and the partial-ring rotating rPET (SUINSA Medical Systems) [6]. Several magnitudes derived from the acquisitions such as intrinsic resolution, scatter fraction, noise equivalent count rate curve and sensitivity were employed for the comparison. NEMA [7] specifications were used, with mouse and rat size cylinders, spheres and line sources as phantoms. The differences obtained between real and simulated data are

S.España is with the Grupo de Física Nuclear at Department of Atomic, Molecular and Nuclear Physics, Complutense University of Madrid, 28040 SPAIN (telephone: +34-913944484, e-mail: samuel@nuclear.fis.ucm.es).

J. L. Herraiz e-mail: joaquin@nuclear.fis.ucm.es.

E.Vicente email: evicente@nuclear.fis.ucm.es.

E. Herrnaz e-mail: eherranz@nuclear.fis.ucm.es.

J.J.Vaquero email: juanjo@mce.hggm.es.

M. Desco e-mail: desco@mce.hggm.es.

J.M.Udias e-mail: jose@nuc2.fis.ucm.es. smaller than $10 \%$, validating PeneloPET as an accurate tool for PET simulations.

Many improvements in the code have been made with respect to previous version [3], with the incorporation of a very realistic signal processing chain and the ability of running simulations in parallel on cluster computers.

\section{PENELOPET}

PeneloPET is a general purpose tool for realistic simulations of PET scanners [3]. PeneloPET consist of two main blocks. The first one deals with PENELOPE routines, generating the input files for the materials and isotopes required for PET and storing interaction paths for each particle. The second block deals with the specific features of the PET system under simulation, such as gamma detectors and electronics. All effects included in the simulation are computed sequentially that means, for example, that random coincidences and pulse pile-up are simulated taking into account the moment in that every particular event occurred during the simulation.

The new version of the code extends and improves its possibilities. Definition of more complex geometries and new materials, the possibility of combining different isotopes and beta spectra for emitted electrons and positrons are some of the new features. Pulse pile-up, random coincidences and dead time are now simulated in more detail; block detector missalignments can now be defined and dynamical acquisition can be simulated. This new version also includes the possibility of running the simulation in parallel mode on cluster of computers. PeneloPET has been also updated with the last version of PENELOPE 2006.

\section{MATERIALS \& METHODS FOR VALIDATION}

All measurements were done in accordance with NEMA NU-2 2001 [7] specifications, adapted to small animal PET scanners. Simulations set-ups were as close as possible to the real acquisitions and the same methods and codes were employed to analyze both real and simulated data.

\section{A. Geometry Definitions}

The eXplore VISTA DR (GEHC) consists of 36 PMT detector modules, each one coupled to a dual layer array of $13 \times 13 \mathrm{LYSO}+\mathrm{GSO}$ scintillation crystals. Each pixel crystal has a section of $1.45 \times 1.45 \mathrm{~mm}^{2}$ and between them there is a white reflector $0.1 \mathrm{~mm}$ thick. Pitch size is $1.55 \mathrm{~mm}$ and the length of LYSO and GSO is 7 and $8 \mathrm{~mm}$ respectively. 36 modules are arranged in two rings of 18 modules with a diameter of $118 \mathrm{~mm}$. 
The rPET (SUINSA Medical Systems) is a rotating scanner formed by 4 PMT detector modules, each one coupled to a single layer array of 30x30 MLS crystals. Pitch size is $1.6 \mathrm{~mm}$ and MLS crystals length is $1.2 \mathrm{~mm}$. Ring diameter is $160 \mathrm{~mm}$.

For the validations against the eXplore VISTA DR (GEHC) scanner we employed the data measured by Yuchuan et al. [5]. In what follows, we describe the methods employed to obtain the validation data for the rPET scanner.

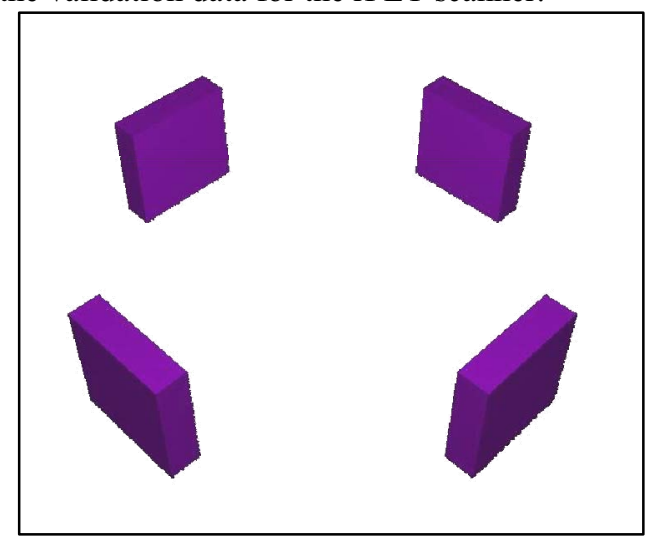

a)

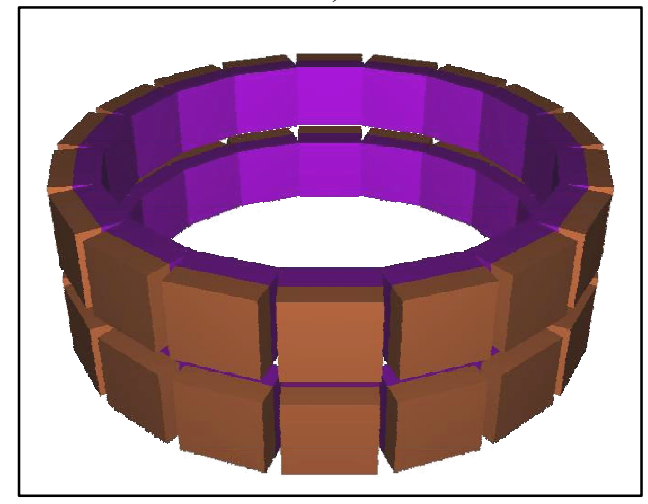

b)

Fig. 1. Schematic drawing of the of the scintillators elements of rPET a) and eXplore Vista b) scanners obtained from the geometry descriptions introduced in the PeneloPET simulations. They were drawn with the application gview3d provided with PENELOPE 2006. The shieldings of the scanners, though employed in the simulations, are not shown here for clarity.

\section{B. Intrinsic Resolution}

An ${ }^{18} \mathrm{~F}$ point source was employed to measure the intrinsic resolution of both systems. The source was placed at the center of the transaxial FOV and shifted in small steps $(\sim 0.3 \mathrm{~mm})$ along the axial direction. Profiles of coincidences among crystals in the same axial row are stored and the average of the FWHM for 13 of these profiles is employed as a measure of the intrinsic resolution.

\section{Scatter Fraction}

One solid plastic cylinder of $2.5 \mathrm{~cm}$ diameter and $6 \mathrm{~cm}$ height, with a hole off-centered by $1 \mathrm{~cm}$ was employed. A line source was placed in the hole filled with low activity of ${ }^{18} \mathrm{~F}$, to minimize the effect of dead time and random and pile-up coincidences. Scatter coincidences (S) are estimated from direct measurements made in the sinogram, by shifting every radial profile so that the maximum of the count profile is placed at the central radial position. Next, all the radial profiles are added and the pedestal, in principle due to random (R) and scatter (S) coincidences is subtracted. It is finally assumed that the true counts $(\mathrm{T})$ are the ones contained in this central region after pedestal removing.

\section{Noise Equivalent Count Rate}

To obtain the NEC rate curve the same phantom described above for scatter fraction estimation is also employed here. As singles information is not available, nor it is possible to employ the delayed coincidence window technique with the rPET scanner, random coincidences were estimated with the same method used to determine the scatter fraction that is, by assuming that the background is composed of scatter plus random coincidences. Once we know the scatter fraction, that is supposed to be constant, we estimated the true coincidence contributions as the one remaining after pedestal subtraction and the random coincidences are calculated as the additional contribution to true and scatter coincidence to yield the total count rate.

\section{E. Sensitivity}

Axial Central Point Sensitivity (ACS) and Axial Sensitivity profiles were also measured. ACS is defined as the efficiency of a point source in the center of the FOV but as placing a true point oruce in the true center of the FOV is extremely difficult, a nearly equivalent ACS measure is usually performed by means of a line source placed at the center of the transaxial FOV. The calculation of the ACS is then computed using the expression $A C S=2 R / A$, where $R$ is the measured coincidence rate and $\mathrm{A}$ is the activity of the line source inside the FOV.

\section{RESULTS}

Here are presented the results of the comparison of the data acquired with the procedure explained above with the ones of the simulations, as well as some figures of merit of the simulations.

\section{A. eXplore Vista DR (GEHC)}

For reference, the rate of simulated particles is presented here for a point source placed at the center of the FOV for this scanner. Simulations run on an AMD Atlhon 3000+ Mhz yielded $6.5 \cdot 10^{3} \mathrm{e}+$ traces simulated per second, when only annihilation photons were simulated.

The intrinsic resolution obtained from experimental data was $1.24 \mathrm{~mm}$, while the results from the simulation was 1.29 $\mathrm{mm}$, that is, there was a slight overestimation. This may be due to the fact that the simulation does not take into account the reflector material that surrounds the crystals and thus the actual crystal size simulated is slightly larger than the real one. 


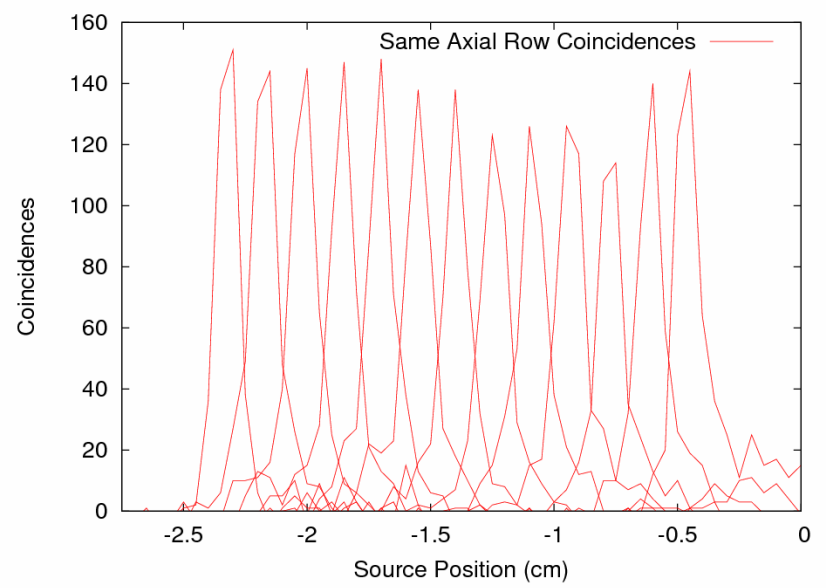

Fig. 2. Simulated profiles for a $22 \mathrm{Na}$ point source employed during intrinsic resolution estimation of Vista scanner.

On table I the results for the estimation of the scatter fraction is presented. Differences between real and simulated values remain below 5\% for both the 250-700 and 400-700 $\mathrm{keV}$ energy windows. For the $100-700 \mathrm{keV}$ energy window, the simulated values are systematically smaller (by about $10 \%$ ) than the real ones, most likely due to the fact that the simulations did not include secondary particle emissions such as X-rays that can contribute at low energies.

TABLE I

SCATTER FRACTION FOR EXPLORE VISTA SCANNER

\begin{tabular}{|c|c|c|c|c|}
\hline \multirow{2}{*}{$\begin{array}{l}\text { Energy Window } \\
(\mathrm{keV})\end{array}$} & \multicolumn{2}{|c|}{ Mouse phantom } & \multicolumn{2}{|c|}{ Rat phantom } \\
\hline & Real & Simulated & Real & Simulated \\
\hline $100-700$ & $33 \%$ & $29 \%$ & $48 \%$ & $42 \%$ \\
\hline $250-700$ & $27 \%$ & $27 \%$ & $37 \%$ & $38 \%$ \\
\hline $400-700$ & $19 \%$ & $20 \%$ & $29 \%$ & $29 \%$ \\
\hline
\end{tabular}

For the validation of the axial profile sensitivity, the detailed model of all the shielding (lead, aluminum, delrim,...) was crucial for the regions at the axial center of each ring and near the edges of the axial FOV.

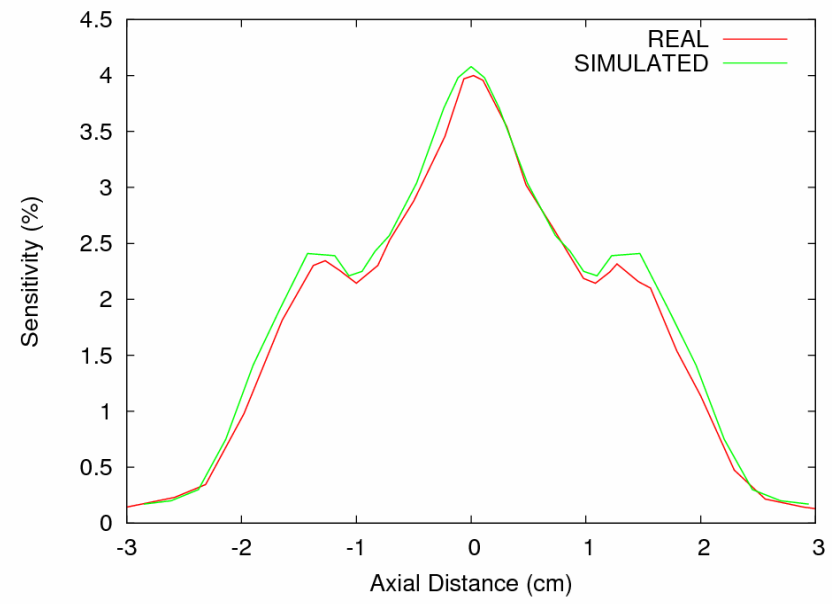

Fig. 3. Axial sensitivity profile of the eXplore VISTA scanner measured with a ${ }^{18} \mathrm{~F}$ point source shifted in small steps along the central axis of the FOV.

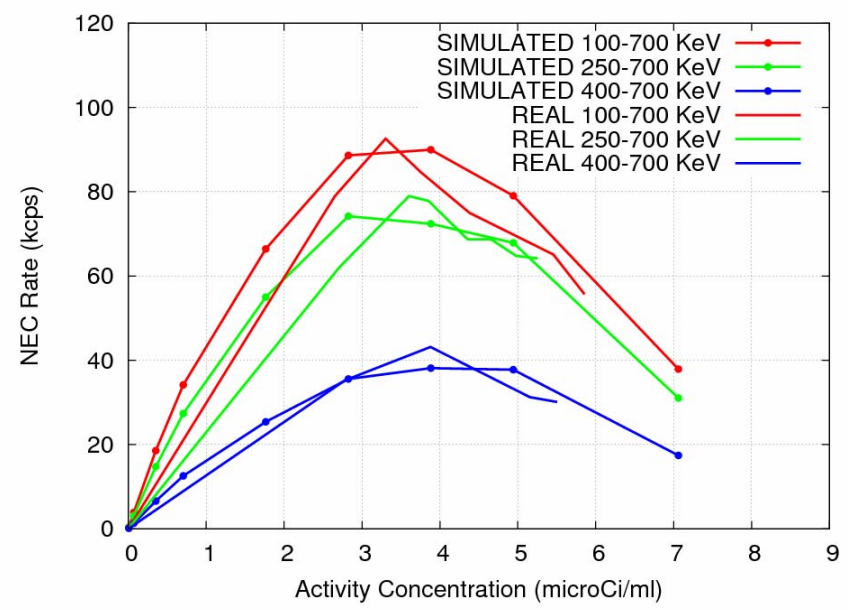

Fig. 4. NEC Rate curves for three energy windows measured for a rat size phantom with the eXplore VISTA scanner. The overall agreement between theory and simulation is good.

In Fig. 4, the NEC rate curves are shown, and a overall good agreement of real and simulated results can be observed.

\section{B. $\quad$ rPET (SUINSA Medical Systems)}

Simulations for a point source in the center of the FOV were performed for this scanner obtaining a simulation rate of $5 \cdot 10^{4}$ $\mathrm{e}+/ \mathrm{sec}$ under the same conditions as previously describe for the Vista case. The increase in simulation speed with respect to the Vista results is due to the simplified detector and shielding geometries of rPET.

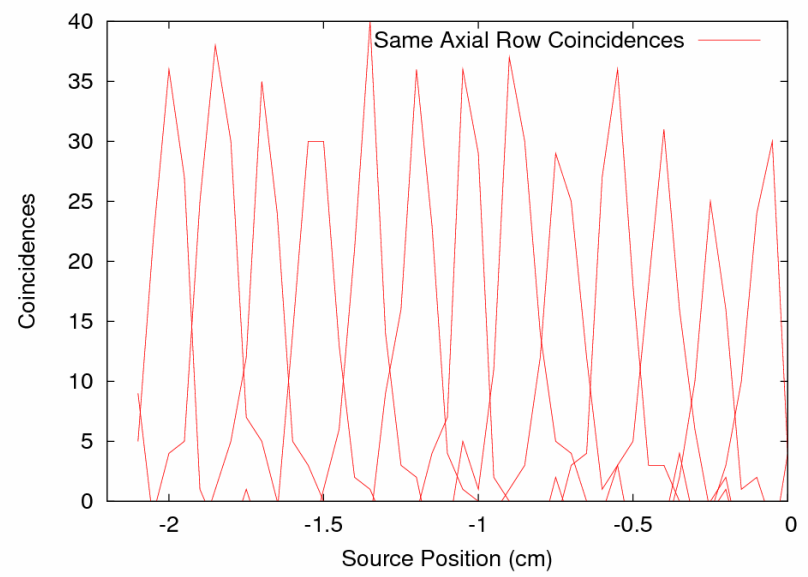

Fig. 5. Simulated profiles for a $22 \mathrm{Na}$ point source employed during intrinsic resolution of rPET scanner.

In Table II the results for some magnitudes obtained for the validation the rPET scanner are shown.

TABLE II

VALIDATION RESULTS FOR RPET SCANNER

\begin{tabular}{ccc} 
& Real & Simulated \\
\hline Intrinsic Resolution (mm) & 1.50 & 1.43 \\
ACS (\%) & 2.1 & 2.0 \\
Scatter Fraction (\%) & 20 & 22 \\
\hline
\end{tabular}


In Fig. 6, the NEC rate curves are shown for the rPET scanner and a good agreement of real and simulated results is obtained.

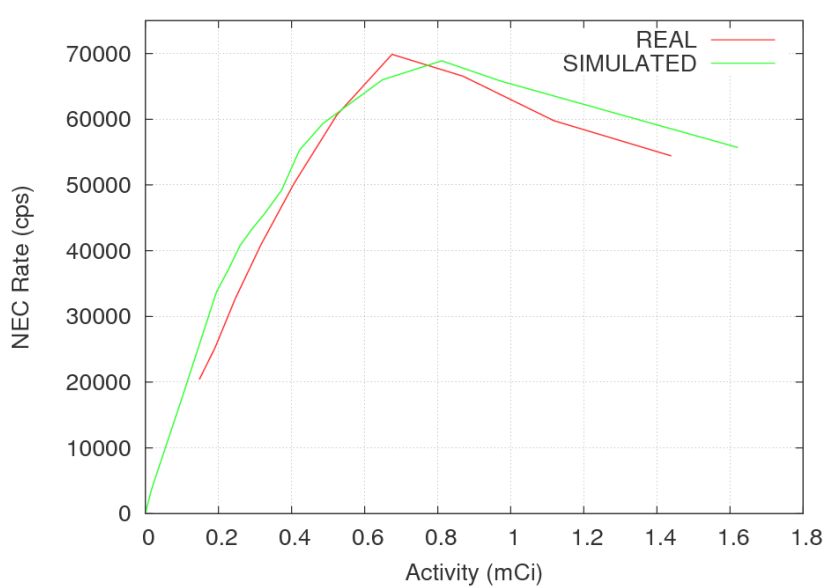

Fig. 6. NEC Rate curve measured with a mouse size phantom for the rPET scanner. These results correspond to the $100-700 \mathrm{keV}$ energy window.

\section{CONCLUSIONS}

This work presents a validation of the new version PeneloPET code against two real scanners. The results indicate that there are only small differences (typically much less than $10 \%$ ) between scanner parameters measured from real acquisitions or simulations. We conclude that PeneloPET is an accurate tool for PET simulations potentially useful for many PET applications such as system response matrix calculations for statistical reconstructions methods [8], development of new PET scanner, validation of correction methods for scatter, random coincidences, pile-up, attenuation, normalization. Future work will include the extension of PeneloPET to SPECT simulations.

\section{ACKNOWLEDGMENT}

This work has been partially funded by projects TEC200407052-C02 of Ministerio de Educación y Ciencia and CDTEAM, program CENIT, Ministerio de Industria, Spain.

\section{REFERENCES}

[1] Harrison R L et al, " Acceleration of SimSET photon history generator module of a public domain simulation system for emission tomography" Conf. Rec. IEEE Nucl. Sci. Symp and Med. Imaging Conf.3 1835-8.

[2] S. Jan et al. , "GATE: a simulation toolkit for PET and SPECT". Phys. Med. Biol. 49 (2004) 4543-4561.

[3] S. Espana, J. L. Herraiz, J. J. Vaquero, M. Desco, and J. M. Udias, "PeneloPET: A MonteCarlo PET simulation tool based on PENELOPE," presented at IEEE Nuclear Science Symposium and Medical Imaging Conference, 2006.

[4] J. Sempau, E. Acosta, J. Baró, J.M. Fernández-Varea, F. Salvat, “An algorithm for Monte Carlo simulation of coupled electron-photon transport", Nucl. Inst. Meth. In Phy. Res. B 132 (1997) 377-390.

[5] Yuchuan W., Seidel J., Tsui B.M.W., Vaquero J.J., Pomper M. G., "Performance Evaluation of the GE Helthcare eXplore VISTA DualRing Small-Animall PET Scanner", J. Nucl. Med. Vol. 47 No. 11 18911900 .
[6] J. J. Vaquero, E. Lage, L. Ricon, M. Abella, E. Vicente, and M. Desco, "rPET Detectors Design and Data Processing," presented at IEEE Nuclear Science Symposium and Medical Imaging Conference, 2005.

[7] National Electrical Manufacturers Association 2001 NEMA Standards Publication NU2-2001: Performance Measurements of Positron Emission Tomographs (Washington DC:NEMA)

[8] J. L. Herraiz, S. Espana, J. J. Vaquero, M. Desco, and J. M. Udias, "FIRST: Fast Iterative Reconstruction Software for (PET) tomography," Phys. Med. Biol., vol. 51, pp. 4547, 2006. Fan-Beam Collimators," Nuclear Science, IEEE Transactions on, vol. 50, pp. 398-404, 2003. 